\title{
Innovative design choices for the Large Synoptic Survey Telescope
}

Victor L. Krabbendam

Cutting-edge technology and novel approaches target transformative scientific return from the next-generation astronomical survey telescope.

The Large Synoptic Survey Telescope (LSST) is a new groundbased telescope designed to address some of today's most compelling questions ${ }^{1}$ in astronomy and high-energy physics. During its planned ten-year survey operation, it will map the visible sky deeply, rapidly, and continuously, producing an archive of hundreds of images covering the entire sky. The same data set can be used to characterize the properties of dark energy, produce nearly instant alerts of serendipitously detected optical transients (such as exploding stars in distant galaxies), discover and provide orbits for potentially hazardous near-Earth objects, and catalog billions of targets with high astrometric precision and to unprecedented photometric depths. By publishing the images, alerts, and catalog products (without any proprietary period), the LSST will transform the way astronomers and high-energy physicists conduct research. This will also enable educators, students, and the general public to actively participate in the process of discovery. The LSST project ${ }^{2}$ includes all phases of this initiative, from building the telescope and camera to developing the data-management system and conducting the ten-year survey.

The telescope design (see Figure 1) is based on a threemirror configuration, with an $8.4 \mathrm{~m}$ (diameter) primary, a $3.4 \mathrm{~m}$ secondary, and $5 \mathrm{~m}$ tertiary mirror, feeding a three-element refractive-corrector camera system to produce a $3.5^{\circ}$ (diameter) field of view with excellent image quality. The $6.7 \mathrm{~m}$ (diameter) effective collecting area and 9.6 square degree field of view offer massive data-collecting capacity, dwarfing any other current or planned astronomical facility.

The fast $f / 1.23$ (focal ratio) optical system allows for a compact overall structure, designed with a first natural frequency of $8.3 \mathrm{~Hz}$ analyzed on its pier and native-base soil. The drives and damping-control system achieve repointing of $3.5^{\circ}$ on the sky - to an adjacent field-in 5s. The survey requires taking a

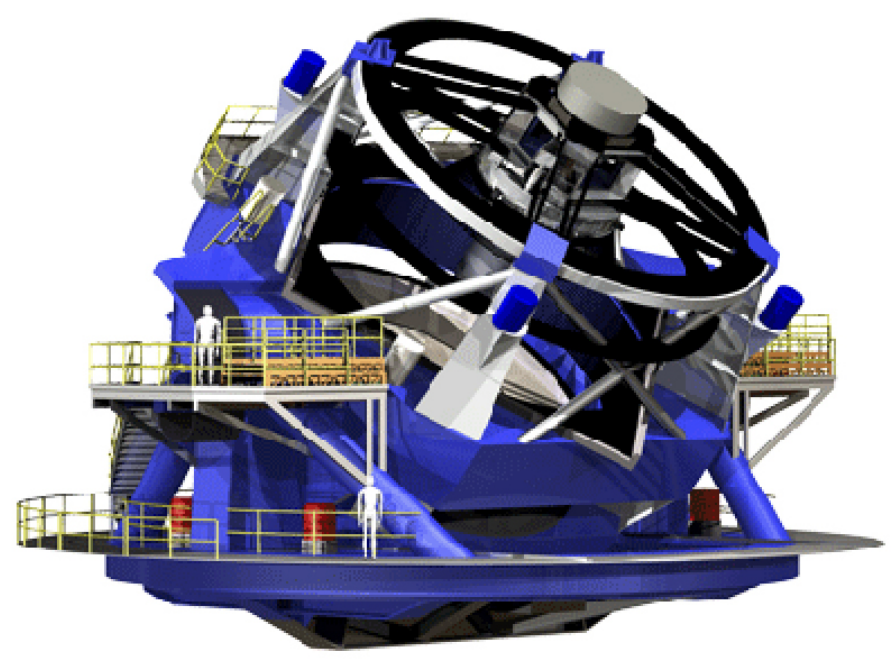

Figure 1. The 300 ton Large Synoptic Survey Telescope (LSST) structure is an elevation-over-azimuth configuration.

pair of $15 \mathrm{~s}$ exposures separated by a $2 \mathrm{~s}$ readout time, followed by a nominal $5 \mathrm{~s}$ slew, for a total of $\sim 40$ s per pointing on the sky. With this cadence, the LSST will image the entire accessible sky every few nights, obtain repeat exposures at frequencies of $15 \mathrm{~s}$, a few tens of minutes, weeks, and years, and provide both a deep coadded image of the entire visible sky and a new movie-like survey of our dynamic universe.

The camera ${ }^{3}$ has a $1.6 \mathrm{~m}$ diameter front lens and two smaller lenses to correct for chromatic aberration before photons reach the $63 \mathrm{~cm}$ diameter focal plane. The focal-plane detector consists of 189 silicon CCDs, each containing $4000 \times 4000$ pixels, with a total of 3.2 billion pixels. Each device is segmented into 16 readout channels to achieve a $2 \mathrm{~s}$ readout time. Five of the six filters are included in the camera body during operation to allow for efficient filter switching during the night. The full operational spectral range spans from 330 to $1070 \mathrm{~nm}$.

The observatory will produce 15 terabytes of raw image data each night that the data-management system must transport,

Continued on next page 


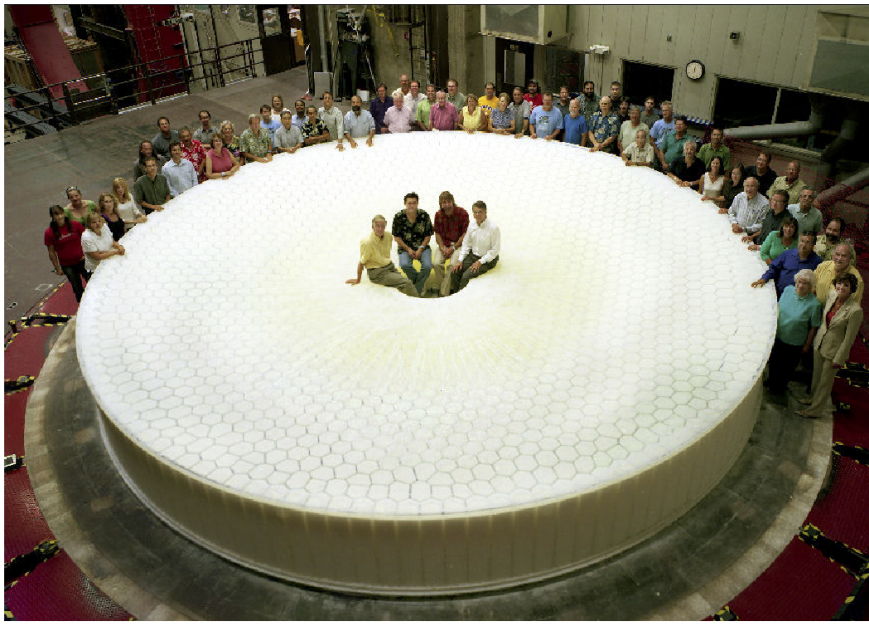

Figure 2. Members of the LSST and Steward Observatory Mirror Laboratory teams gather around the recently cast LSST primary mirror.

process, and archive continuously. ${ }^{4}$ The system will also broadcast alerts of any optical transient within one minute of any relevant observation. The National Center for Supercomputing Applications in Illinois will host the archive center, which will grow to roughly 60 petabytes. Data-access centers will handle data distribution.

Federal funds are currently supporting key design and development activities. Private funding has enabled construction of the telescope mirrors. The primary and tertiary mirrors are constructed at the University of Arizona's Steward Observatory Mirror Laboratory using cast-borosilicate mirror technology. The proximity of the primary and tertiary surfaces allows fabrication of both mirrors from a single substrate (see Figure 2). ${ }^{5}$ This is the first time that two optical surfaces are polished into a single mirror on this giant scale. This approach offers significant advantages for reducing the number of degrees of freedom during operational alignment and to improve structural stiffness of the otherwise annular primary mirror. Constructing the $3.4 \mathrm{~m}$ diameter secondary mirror was also recently started with private funding. Corning Inc. will manufacture the near-net-shape substrate using ultralow-expansion (ULE) ${ }^{\mathrm{TM}}$ thin-meniscus mirror technology.

Innovative approaches and technology are used in each area of the project to address key engineering challenges. The LSST will be sited on Cerro Pachón in northern Chile (see Figure 3), where it will benefit from excellent observing conditions. The project team ${ }^{6}$ is planning a five-year construction phase, which can begin as soon as federal funding has been authorized.

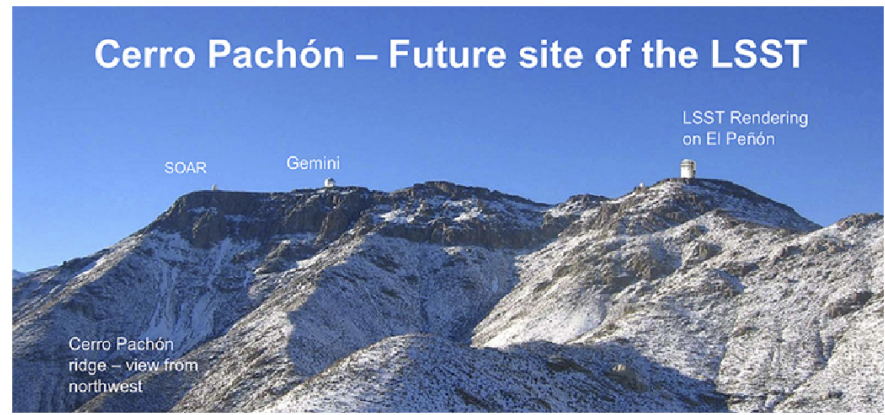

Figure 3. Cerro Pachón, where LSST has recently completed the environmental-impact process and been granted building permits. SOAR: Southern Astrophysical Research telescope.

The LSST research and development effort is funded in part by the National Science Foundation under scientific program order No. 9 (AST-0551161) through cooperative agreement AST-0132798. Additional funding comes from private donations, 27 LSST-consortium institutional members, and in-kind support from Department of Energy laboratories.

\section{Author Information}

\section{Victor L. Krabbendam}

LSST Project

National Optical Astronomy Observatory

Tucson, AZ

Victor Krabbendam is the LSST project's deputy project manager, responsible for telescope and site aspects. He previously served on the project teams developing the Southern Astrophysical Research (SOAR) and Hobby Eberly telescopes.

\section{References}

1. Ž. Ivezić et al., LSST: from science drivers to reference design and anticipated data products, arXiv:0805.2366v1, 2008. http://arxiv.org/abs/0805.2366

2. V. L. Krabbendam, The Large Synoptic Survey Telescope concept design overview, Proc. SPIE 7012, p. 701205, 2008. doi:10.1117/12.790253

3. K. Gilmore et al., The LSST camera overview: design and performance, Proc. SPIE 7014, p. 70140C, 2008. doi:10.1117/12.789947

4. J. Kantor et al., High performance astronomical data communications in the LSST data management system, Proc. SPIE 7019, p. 701919, 2008. doi:10.1117/12.790194

5. H. M. Martin et al., Manufacture of a combined primary and tertiary mirror for the Large Synoptic Survey Telescope, Proc. SPIE 7018, p. 70180G, 2008. doi:10.1117/12.789959

6. http://www.lsst.org LSST project website. 